\title{
The Design and Simulation a Subsystem under Electromagnetic Interference to Enhance the System's Performance
}

\author{
Milad Daneshvar, Naser Parhizgar, and Homayoon Oraizi
}

\begin{abstract}
High-speed digital boards should be able to have integrity validation credentials within the framework of the specified criteria. In order to improve the simulation results, the package designer should consider the management of the radiation level at the board, which will reduce the time and cost of implementing the design and manufacturing process. In order to achieve this goal, the integrity (signal, power, heat and data) is of great importance. With the modeling and analysis of the transmission line in one of the near paths of clock to reduce electromagnetic interference, good results can be obtained from the correct operation of the board. Due to the nature and location of the components and the complexity of the input signals, it also increases reliability of components and the tolerance of electromagnetic interactions, to evaluate the performance of the subsystem under the influence of radiation, various practical tests on the range and results was recorded.
\end{abstract}

Index Terms - Signal Integrity; Power Integrity; Data Inetgrity; Termal Integrity; Electromagnetic Interference

\section{INTRODUCTION}

In the past, the frequency of the systems did not exceed a few $\mathrm{MHz}$, and single- or double-layer PCBs were used solely for laying the elements and communicating between them. Also, how the elements and paths were connected and positioned had no effect on system performance. But nowadays, with the increase of frequency up to several $\mathrm{GHz}$, new phenomena may occur that if the board is not properly designed and not familiar with the principles needed in this field, it will cause system malfunction. Therefore, it is necessary to pay more attention to the design and routing of signals $[1,4]$. The problem of thermal integrity causes the voltage difference on the Grand. The voltage difference on the Grand every 10 degrees above 100 degrees halves the average failure rate and every 10 degrees causes 4 percent ohms of copper to deteriorate, which is a destructive phenomenon. The thermal integrity causes power integrity because it actually warms a part of the circuit and raises the $\mathrm{ohm}$ of the part, causing this problem, and if the board is expanded, some of the base pieces may be removed from the

Published on November 3, 2019

Milad Daneshvar, Department of Electrical Engineering, Shiraz branch, Islamic Azad University, Shiraz, Iran.

(email: milad_daneshvar@yahoo.com)

Naser Parhizgar, Department of Electrical Engineering, Shiraz branch, Islamic Azad University, Shiraz, Iran. (e-mail:naserpar@yahoo.com)

Homayoon Oraizi, Department of Electrical Engineering, Iran University of Science and Technology (IUST), Tehran, Iran.

(e-mail: h_orayzi@iust.ac.ir) board. Inductor and current are always two major problems in conflict. To solve the problem of power integrity we need to increase the thickness of copper. Optimize the via location and add copper where possible (because it is incorrect and may cause signal integrity problems and cause impedance mismatch). The best thing is to have one layer per voltage, which is, of course, expensive and makes it difficult to analyze power integrity [2,3]. It is the art of the designer to design the least hardware and software. The simplest problem solved in the board is the signal integrity associated with the signal itself, and we have that problem if we don't have another signal. In analyzing signal integrity, we need to know the IC inputs and outputs. The integration of the signal resulting from the return losses gives the integration that is visible in the eye diagram. If the eye diagram is open it is better because the data is more reliable. When we solve the signal integrity problem, the eye closes [5]. Data integrity is the speed of movement of electrons along the path. To solve the data integrity problem, all ICs output sheets must be of equal length, and if we do not solve the problem in the circuit we have a timing problem which is very important in digital circuits $[6,7]$.

\section{DESIGN AND SIMULATION OF RADIATION-AFFECTED RANGE}

Modeling and analysis of the transmission line One of the nearest clock paths to reduce EMI, in the vicinity of flowthrough components with Hyperlinx software, was simulated and the results were recorded.

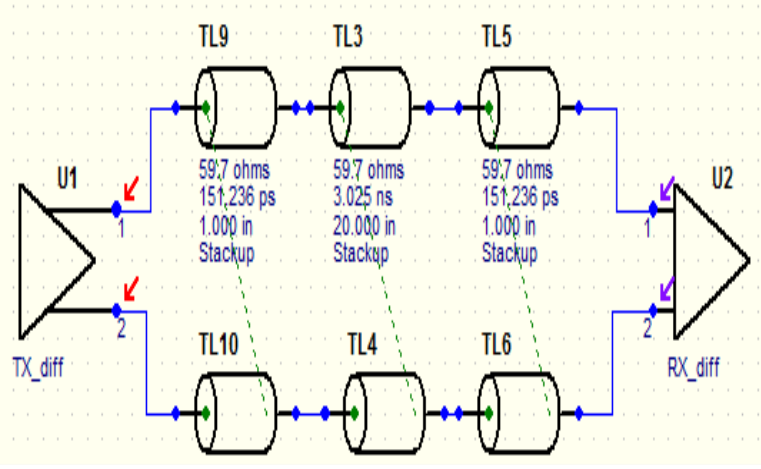

Fig. 1 Modeling and analysis of the test subsystem transmission line 
A. Characteristics of the subsystem board layers under test The board is 8 layers. The full specifications of the board are shown below:

Top: 1.2 mil Cu Signal $Z_{0}=96 \Omega$

Middle: 12 mil FR4

Middle: 1.2 mil $\mathrm{Cu}$ shield GND

Middle: 12 mil FR4

Middle: 1.2 mil Cu shield VCC

Middle: 12 mil FR4

Middle: $1.2 \mathrm{mil} \mathrm{Cu}$ shield GND

Bottom: $1.2 \mathrm{mil} \mathrm{Cu}$ Signal $\mathrm{Z}_{\mathrm{o}}=96 \Omega$

Normally the width of Trace is 6 milliseconds. The inner layer impedance with the Trace nominal width $(6 \mathrm{mil})$ is $71.2 \Omega$. In this board, analogue and digital grounds are interconnected. These changes have been made to improve the radiation emission at the frequency range (150KHZ25MHZ). Initially studies were made on the types of boards with similar applications and related reference designs and a number of related references designs were downloaded from the Internet. This information was added to the data collected and based on the reverse engineering of the board. Initially, this was done on a board-scanned photo and the paths and components were identified during the process. MARKETING Many components were identified using the SMD code books and the regulator chip was identified by examining all of the FairChild regulator chips and their reference-layouts. Since all the references were designed using Cadence Allegro and the features of this tool for drawing schematic and board were one of the best options, the board was implemented with this tool. Schematic circuit recovery was the hardest part of the job. The schematic circuit is designed to operate at $5 \mathrm{~V}$ and $3.3 \mathrm{~V}$ by the regulator. If you assemble the resistor $0 \mathrm{ohm} \mathrm{R} 1$ and remove the $\mathrm{D} 1$ diode, the circuit will work at $3.3 \mathrm{~V}$, and if you assemble the D1 diode and remove the R1 resistor, the circuit will work at $5 \mathrm{~V}$ input. Relevant documents indicate that this board is the manufacturer's Revision2. Due to the importance of tolerating the harsh conditions for the systems processing center, changes in this circuit include the addition of a $10 \mathrm{nf}$ capacitor at the base of the InterPoint IC input, the addition of a relatively large capacitor and an ESD shielding diode at the $5 \mathrm{~V}$ input of the power supply and addition. Some resistance to the boot circuitry has been applied to the design considerations for design considerations. Due to the limited range of sizes and the fact that we had to increase the number of pieces to make sure the circuit functioned, we reduced the range to standard size. In other words, we increased the width of the board by $5 \mathrm{~mm}$ and used the standard pattern as the circuit board. In the implementation process, due to the interconnection between the internal points of the circuit, the routing of the board was very time consuming and almost entirely manual. Of course, the automated engines included in the software also played a big role in helping the route. It should be noted that several different versions of the board were made with different layers and layouts, but this version eventually provided the conditions for passing the tests. This IC uses two ICs, the routing conditions of which are in accordance with the Agilent standard. The circuit has 7 pairs of high-speed differential connectors, $150 \mathrm{MHz}$ line, and a DDR2 memory chip that has up to $200 \mathrm{MHz}$ data exchange with the processor and the DDR2-connected pins should typically have a peak time of about half a nanosecond or less. Of course, in the 2,500 pages of the controller chip document there is no mention of this, and we have to cite for example the layout guide document for this family of chips the maximum length allowed for data paths, addresses, and controls connected to the processor. Consider $2.25 \mathrm{~cm}$ or 885 milliseconds to avoid the problem of signal integrity. In practice, if the path length for the FR4 chip is less than $0.3 *$ rise-time $* 7.5$ in centimeters, there is no need to add end-toend reflection correction [3].

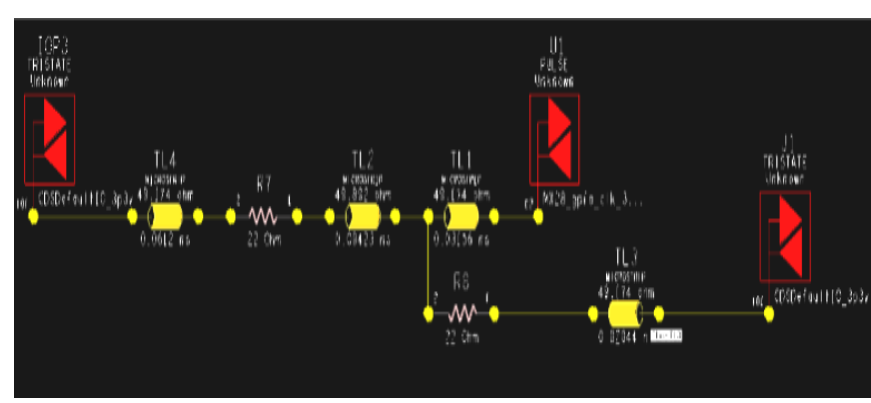

Fig. 2 The $50 \mathrm{MHz}$ NET Clock transmission line

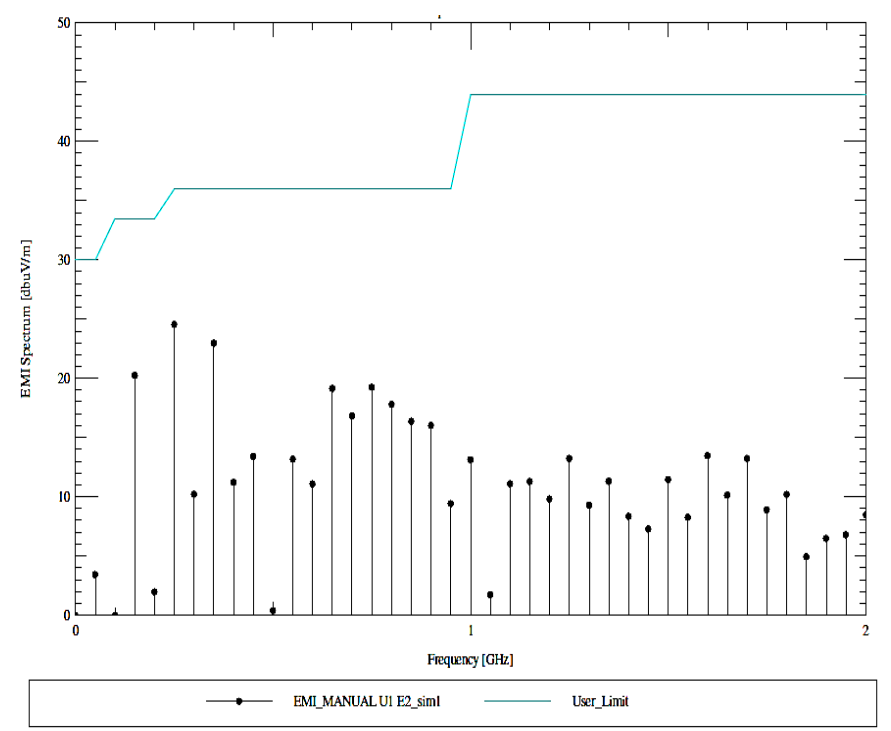

Fig. 3 Clock radiation with a frequency of $50 \mathrm{MHz}$ is acceptable in the FCC Class B standard. 


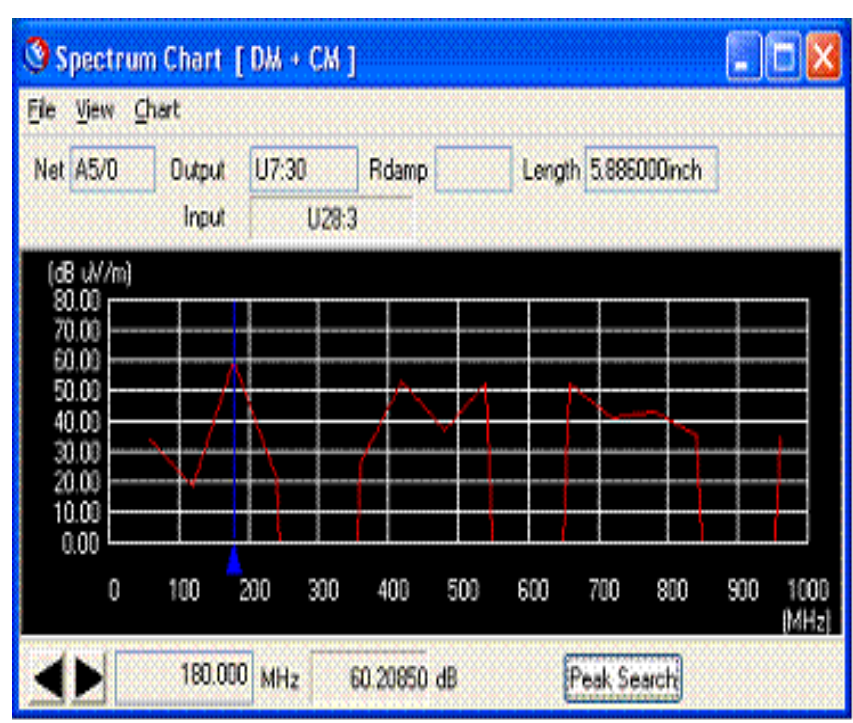

A

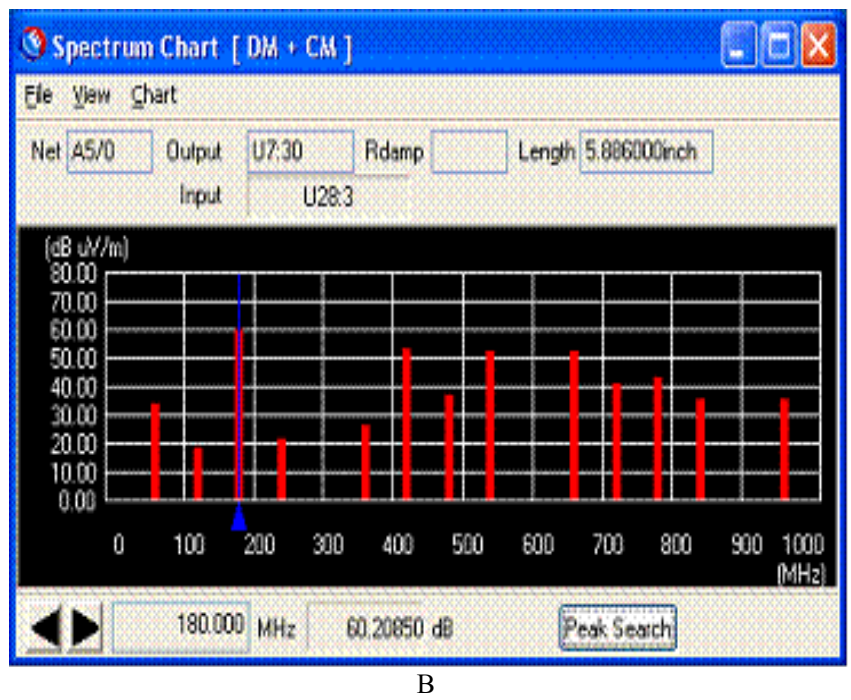

Fig. $4 \mathrm{~A}$ and $\mathrm{B}$ is shown the signal spectrum diagrams

\section{B. Technical Specifications Board Testing Affected by RF Signal}

The following tests were applied to the board and results were recorded to evaluate the performance of subsystems affected by radiation.

\section{Ohm's path test}

To perform this test by setting the specified paths from the input to the outputs of the board, the path ohmic resistance was measured by the LRCMETER with a accuracy of 0.1 millimeter.

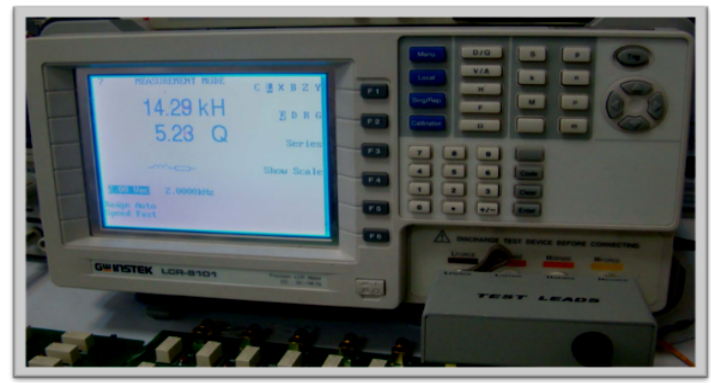

Fig. 5 View of the LCR meter
The steps of this measurement are as follows:

\section{Ohmic signal path test}

The machine connects to the circuits via the RG 178 cable with an input probe.

Table I

The results of the ohmic signal path test results

\begin{tabular}{|c|c|}
\hline Input to output & Path resistance(ohm) \\
\hline $1-1$ & 1.572 \\
\hline $1-2$ & 1.52 \\
\hline $2-1$ & 1.381 \\
\hline $2-2$ & 1.54 \\
\hline
\end{tabular}

\section{Ohmic test of power supply path}

The device connects to the circuits via the RG 178 cable with an input probe.

Table II

Results of ohmic test of power supply path

\begin{tabular}{|c|c|}
\hline Input to output & Path resistance(ohm) \\
\hline $1-1$ & 1.47 \\
\hline $1-2$ & 1.274 \\
\hline $1-3$ & 1.185 \\
\hline $1-4$ & 1.392 \\
\hline
\end{tabular}

\section{Path capacitance}

For this test, the capacitance of the path is measured by the LRC METER device by defining the paths from the input to the output. The device connects to the circuits via the RG 178 cable with an input probe.

Table III

Path Capacity Test Results

\begin{tabular}{|c|c|c|}
\hline NO. Board & Input to output & Path capacitance \\
\hline 01 & unilateral & $5 \mathrm{PF}$ \\
\hline
\end{tabular}

\section{Phase shift test}

For this test, the phase difference was measured by digital oscilloscope (capable of measuring phase difference) by defining the paths from input to output by applying a sine signal with a specified amplitude. The input is connected to the circuits via the RG 178 cable through the generator function $(\mathrm{RL}=50 \mathrm{ohm})$.

Table IV

Phase Shift Test Results

\begin{tabular}{|c|c|c|c|}
\hline Amplitude & Frequency & $\begin{array}{c}\text { Measured phase } \\
\text { shift }\end{array}$ & $\begin{array}{c}\text { Max. phase } \\
\text { shift }\end{array}$ \\
\hline $13 \mathrm{dBm}$ & $2.2 \mathrm{MHz}$ & 47.52 & 52.8 \\
\hline
\end{tabular}

To measure phase difference less than $2 \mathrm{MHz}, 1 \mathrm{MHz}$ load resistance is required which is not currently available. The measured PSM values in this test correspond to the values given in the technical specifications.

Max phase shift $=2.4 * \mathrm{f} * 10^{\wedge}-5, \mathrm{RL}=50 \mathrm{ohm}$

\section{Frequency response test}

It is obtained by a fixed start and end frequency and by a fixed input amplitude at different stages of operation.

Start Frequency $=9 \mathrm{KHZ}$ 
Stop Frequency $=40 \mathrm{MHZ}$

Input Amplitude $=-13 \mathrm{dBm}$

\section{Hold current}

To perform this test, each system output is connected to a load of $2.4 \Omega$ and by applying a voltage of $12 \mathrm{~V}$, a current of 2.8 amp. passes through the circuit. It should be noted that this test took 10 minutes. Also, there was no change in impedance profile after performing this test.

\section{THE RESULTS OF DIFFERENT SIGNALS FROM THE RADIATION BOARD}

The board was tested under radiation and the results were recorded in the corresponding test log.

Table V

Phase Shift Test, Capacity and path Resistance LINE RESISTANCE \& CAPACITY \& PHASE SHIFT TESTS SUPPLY - CONNECTION

\begin{tabular}{|c|c|}
\hline \multicolumn{2}{|c|}{ SUPPLY - CONNECTION } \\
\hline Path resistance value & Path capacitance value \\
\hline $550 \mathrm{~m} \Omega$ & 0 \\
\hline \multicolumn{2}{|c|}{ SIGNAL - CONNECTION } \\
\hline Path resistance value & Path capacitance value \\
\hline $780 \mathrm{~m} \Omega$ & 0 \\
\hline \multicolumn{2}{|c|}{ Phase Shift } \\
\hline Frequency & Phase difference \\
\hline $3 \mathrm{MHz}$ & $38^{\circ}$ \\
\hline
\end{tabular}

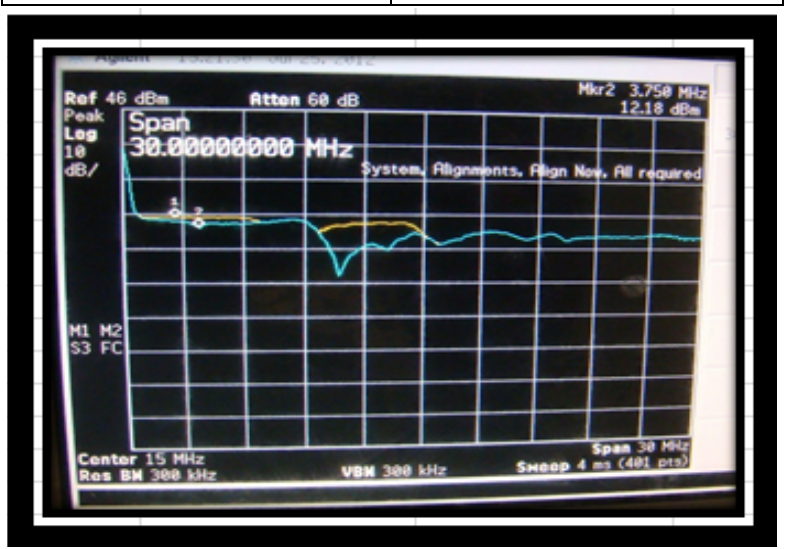

Fig. 6 Frequency response of the subsystem under radiation

\section{CONCLUSION}

Experimental design rules have been used to reduce thermal loss and to comply with electromagnetic compatibility standards. It should be noted that to fully evaluate the system, all the necessary parameters such as thermal, power, signal and data integrity at different temperature conditions and under the influence of radiation mode interference, and all design requirements must be observed and then final modeling, results are simulated in Hyperlinx software and compared to practical results to ensure system performance, which can be largely estimated using relative allocation methods.

\section{REFERENCES}

[1] J. Preibisch, X. Duan, C. Schuster, "Corrections to 'An Efficient Analysis of Power/Ground Planes with Inhomogeneous Substrates Using the Contour Integral Method "”, IEEE Transactions on Electromagnetic Compatibility, vol. 59, no. 3, page 1000, June 2017.

[2] C. Schuster, "Ensuring Signal and Power Integrity for High-Speed Digital Systems “, IEEE International Conference on Consumer Electronics (ICCE), Berlin, Germany, September 6-9, 2016.

[3] C. Schuster, "Signal Integrity Engineering for High-Speed Links ", Global University Lecture at the IEEE Symposium on Electromagnetic Compatibility, Dresden, August 16-22, 2015.

[4] C. Schuster, "Using the Contour Integral Method for Solving Large EMC Problems on Printed Circuit Boards ", Workshop on Computational Electromagnetics and Multiphysics Methods for Characterizing Complex EMC/EMI Effects (WS8) at the IEEE Symposium on Electromagnetic Compatibility, Dresden, August 16$22,2015$.

[5] Cats O, Jenelius E. Planning for the Unexpected: The Value for Reserve Capacity for Public Transport Network Robustness. Transportation Research Part A: Policy and Practice, 2015.

[6] Lundstedt H, Persson T, Andersson V. The extreme solar storm of May 1921: observations and a complex topological model. Annales Geophysicae, 33, 109-116, 2015.

[7] Alderson DL, Brown GG, Carlyle WM. Operational Models of Infrastructure Resilience. Risk Analysis, 2015; 34(4):562-586.

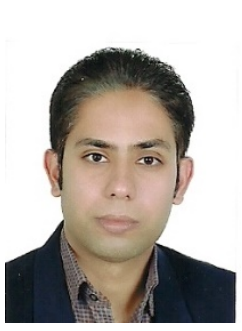

Milad Daneshvar was born in Shiraz, Iran on May 5, 1984. He received B.S. degree from the Shiraz University of Technology, Shiraz, Iran and M.S degree from the Boushehr Branch, Islamic Azad University, Boushehr, Iran and ph.D. Condidate from the Shiraz Branch, Islamic Azad university,Shiraz, Iran, all in Electrical Engineering, in 2007, 2013 and 2018 respectively. He has translated several textbooks in Farsi. He has authored over 40 papers in Electrical Engineering conferences in Iran. In 2016, he was as a reviewer at the 6th.world congress on electrical engineering, computer science and information technology, Barcelona, Spain and In 2015 at the 2nd. International Congress of Electrical Engineering,Tehran, Iran. His major research interest include Electromagnetic Engineering, Signal Processing.

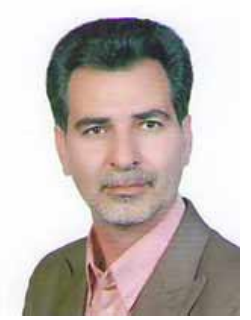

Naser Parhizgar was born in Shiraz, Iran on October 24, 1968. He received B.S. and M.S degrees from Shiraz University,Shiraz, Iran and ph.D. degree from the Iran University of Science and Technology(IUST), Tehran, Iran, all in Electrical Engineering, in 1992, 2007 and 2013 respectively. In 2010, he joined the Department of Electrical Engineering Shiraz Branch, Islamic Azad University, Shiraz, Iran. He was as a Distinguished researcher in Najafabad Branch, Islamic Azad University, Isfahan, Iran, 2007 and Fars Science and Research Branch, Islamic Azad University,Shiraz,Iran, 2011. His major research interest include Communication Engineering, Signal Processing, Aray Processing, RADAR

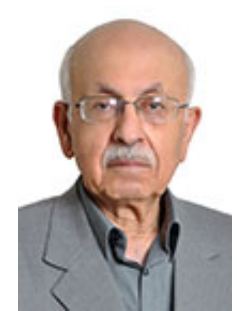

Homayoon Oraizi(SM) received B.E.E. degree from American University of Beirut, Lebanon, in 1967, M.Sc. and ph.D. degrees in electrical engineering from Syracuse University, Syracuse, NY, in 1969 and 1973, respectively. From 1973 to 1974, he was at Toosi University of Technology, Tehran, Iran. From 1974 to 1985 , he was with the Communication Division, Iran Electronics Industries, Shiraz, Iran. In 1985, he joined the Department of Electrical Engineering, Iran University of Science and Technology, Tehran, Iran, where he is a Full Professor of electromagnetic engineering. He has authored and translated several textbooks in Farsi. He has authored or coauthored over 300 papers in international journals and conference. In 2006, he was elected an exemplary nation wide university professor in Iran. He is an Invited Professor of the Electrical Engineering Group, Academy of Sciences of Iran, and is listed as an elite engineer by the Iranology Foundation. 